\title{
Ovário-histerectomia vídeo-assistida com único portal em cadelas: estudo retrospectivo de 20 casos
}

\author{
Single-port video-assisted ovariohysterectomy in bitches: retrospective study of 20 cases
}

\section{Marco Augusto Machado Silva ${ }^{\mathrm{I} *}$ Priscila Andréa Costa dos Santos Batista ${ }^{\mathrm{I}}$ Franco Metzker Pogianni Miguel Ladino Silva ${ }^{I}$ Marina Salles Munerato ${ }^{I}$ Fabíola Neiderauer Flores $^{I}$ Paula Araceli Borges ${ }^{I}$ Alexandre Pinto Ribeiro ${ }^{I}$ Newton Nunes ${ }^{I}$ Gilson Hélio Toniollo}

\section{RESUMO}

$O$ objetivo do presente estudo foi avaliar retrospectivamente as 20 primeiras ovário-histerectomias vídeoassistidas com único portal (SPVA-OSH), realizadas por um cirurgião não proficiente nessa técnica. Vinte cadelas foram submetidas à laparoscopia para SPVA-OSH, com o auxílio de um endoscópio de $10 \mathrm{~mm}$, com canal de trabalho de $5 \mathrm{~mm}$, inserido por um trocarte, posicionado na região pré-púbica, $e$

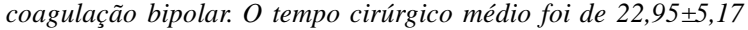
minutos. Os cinco primeiros procedimentos consumiram mais tempo cirúrgico do que os demais. A abordagem aos pedículos ovarianos direito e esquerdo foram as etapas que apresentaram maior tempo de execução. Houve complicação leve ou moderada em seis $(30 \%)$ das 20 cadelas operadas. As complicações foram: hemorragia leve ou moderada em um dos pedículos ovarianos em quatro (20\%) cadelas $e$ hemorragia grave, devido à punção do baço com agulha de Veress em dois (10\%) animais. A SPVA-OSH foi realizada em cadelas por um cirurgião no início da curva de aprendizado, sem complicações maiores.

Palavras-chave: ovário-histerectomia, laparoscopia, caninos, técnica cirúrgica.

\section{ABSTRACT}

The aim of the present study was to evaluate retrospectively the first 20 cases of single-port video-assisted ovariohysterectomy (SPVA-OSH) performed by an inexperienced surgeon. Twenty bitches submitted to SPVA-OSH were assessed. A $10 \mathrm{~mm}$ operative rigid endoscope with one 5 mm working channel and bipolar coagulation was used. The trocar was positioned in the prepubic area of the abdomen. Surgical time and complication rates were assessed descriptively. Mean surgical time was of $22.95 \pm 5.17$ minutes. Mild or moderate complications were present in six (30\%) out of 20 bitches that were operated. The first five surgical procedures spent more time to be performed than the others. The longest transoperative momentum time of execution was spent in the approach to the left and right ovarian pedicles. The complications were: mild or moderate hemorrhage from one of the ovarian pedicles in four bitches (20\%) and severe hemorrhage due to accidental puncture of the spleen with Veress needle in two animals (10\%). SPVA-OSH was performed in bitches by an inexperienced surgeon, without severe complications.

Key words: ovariohysterectomy, laparoscopy, canines, surgical technique.

\section{INTRODUÇÃO}

A ovário-histerectomia $(\mathrm{OSH})$ é um dos procedimentos cirúrgicos mais realizados na rotina de clínicas e hospitais veterinários, cujas principais indicações são: controle da natalidade, estros prolongados, prevenção de tumores mamários, presença de parto patológico e tratamento de enfermidades ovarianas, uterinas e vaginais (HOWE, 2006).

Com o advento das técnicas cirúrgicas minimamente invasivas, a castração por cirurgia laparoscópica vem ganhando popularidade por apresentar vantagens como menor trauma tecidual, manipulação visceral objetiva, menos hemorragia ou mesmo ausência de sangramento, menos dor pós-

'Departamento de Clínica e Cirurgia Veterinária, Faculdade de Ciências Agrárias e Veterinárias (FCAV), Universidade Estadual Paulista (UNESP), Via de Acesso Prof. Dr. Paulo Donatto Castelane, s/n, 14.884-900, Jaboticabal, SP, Brasil. E-mail: silvamam@gmail.com. *Autor para correspondência. 
operatória e período de convalescência mais curto, em comparação com os procedimentos cirúrgicos tradicionais (DEVITT et al., 2005; HANCOCK et al., 2005; VAN NIMWEGEN \& KIRPENSTEIJN, 2007; FREEMAN et al., 2010).

A ovário-histerectomia vídeo-assistida com único portal (SPVA-OSH) constitui uma das modalidades de esterilização minimamente invasivas (GOWER \& MAYHEW, 2008). Os procedimentos vídeo-assistidos envolvem uma etapa intracavitária e outra extra-corpórea (FREEMAN, 1999). Apesar de ser considerado um procedimento minimamente invasivo simples e de rápida execução (GOWER \& MAYHEW, 2008), a SPVA-OSH apresenta alguns riscos ao paciente, sobretudo quando o cirurgião não é proficiente. Poucos estudos foram realizados com enfoque na curva de aprendizado da OSH laparoscópica ou vídeoassistida.

Assim sendo, com este trabalho, objetivouse avaliar retrospectivamente os 20 primeiros casos de SPVA-OSH realizados por um cirurgião não proficiente em procedimentos laparoscópicos, realizados por único portal de acesso, com principal enfoque no tempo cirúrgico e na taxa de complicações cirúrgicas.

\section{MATERIAL E MÉTODOS}

Foram utilizadas 20 cadelas clinicamente hígidas, sem raça definida (S.R.D.), em anestro à colpocitologia, pesando entre 8 e $18 \mathrm{~kg}$ (média $11,2 \pm 5,4 \mathrm{~kg}$ ), encaminhadas pela Associação Protetora dos Animais (APA) do município de Jaboticabal - SP. Os animais foram selecionados mediante exame clínico geral, ginecológico, hemograma, proteínas totais, glicemia, ALT e creatinina, sendo recrutados apenas aqueles que apresentaram normalidade em todos os exames.

As cadelas foram submetidas a jejum hídrico e alimentar de, aproximadamente, 12 horas e tricotomia ampla do abdômen. Os animais foram pré-medicados com associação de cloridrato de clorpromazina $\left(1 \mathrm{mg} \mathrm{kg}^{-1}\right.$, IM) e sulfato de morfina $\left(0,5 \mathrm{mg} \mathrm{kg}^{-1} \mathrm{IM}\right)$. O protocolo anestésico constou de propofol na dose média de $5 \mathrm{mg} \mathrm{kg}^{-1}$ (IV, até a abolição do reflexo laringotraqueal) para indução anestésica e isoflurano, vaporizado em oxigênio a 100\% para manutenção da anestesia. A profundidade anestésica foi controlada com auxílio de monitor de índice bispectral (BIS), mantendo-se os valores entre 50 e 60. A monitoração dos demais parâmetros constou de oximetria de pulso $\left(\mathrm{SpO}_{2}\right)$, capnometria $\left(\mathrm{EtCO}_{2}\right)$, pressão arterial sistólica, diastólica e média, hemogasometria arterial e temperatura retal.

Todos os procedimentos cirúrgicos foram realizados pelo mesmo cirurgião e auxiliar, aptos a realizar procedimentos laparoscópicos, porém sem proficiência em cirurgias realizadas por único portal. Após antissepsia do campo operatório com clorexidine tópico, seguido por álcool 70GL e clorexidine novamente, estabeleceu-se o pneumoperitônio com $\mathrm{CO}_{2}$ por meio de abdominocentese, com agulha de Veress na região hipocôndrica direita. Em seguida, o abdômen foi insuflado com fluxo de $2 \mathrm{~L}$ minuto $^{-1}$, mantendo-se pressão intraperitoneal de $10 \mathrm{mmHg}$. Posteriormente, realizou-se inserção às cegas de um trocarte de $11 \mathrm{~mm}$ na região pré-púbica (Figura 1A).

Após o acesso à cavidade abdominal, inseriu-se o endoscópio rígido ${ }^{\mathrm{a}}$ de $10 \mathrm{~mm}$ com canal de trabalho para instrumentos de $5 \mathrm{~mm}$ (Figura 1B) para inspeção inicial da cavidade abdominal. $\mathrm{O}$ ovário esquerdo foi localizado e apreendido com pinça de Babcock de $5 \mathrm{~mm}$ de diâmetro e $42 \mathrm{~cm}$ de comprimento, inserida pelo canal de trabalho do endoscópio. O corno uterino esquerdo foi então suspenso até a musculatura abdominal para aplicação de sutura de reparo transparietal com fio de náilon 0 (Figura 2A), permitindo a exposição do complexo arteriovenoso ovariano (CAVO) (Figura 2B). Posteriormente, a pinça de apreensão foi substituída pela pinça de coagulação bipolar e corte simultâneos ${ }^{\mathrm{b}}$, de $5 \mathrm{~mm}$ de diâmetro e $42 \mathrm{~cm}$ de comprimento e seccionou-se o CAVO após diatermia

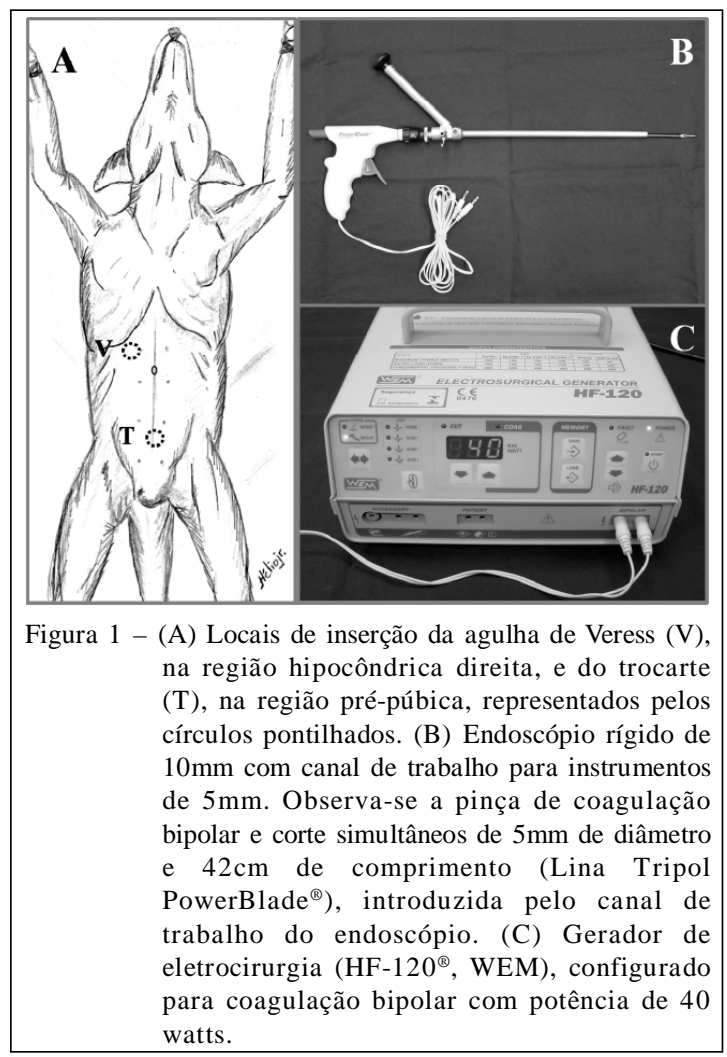

Ciência Rural, v.41, n.2, fev, 2011. 


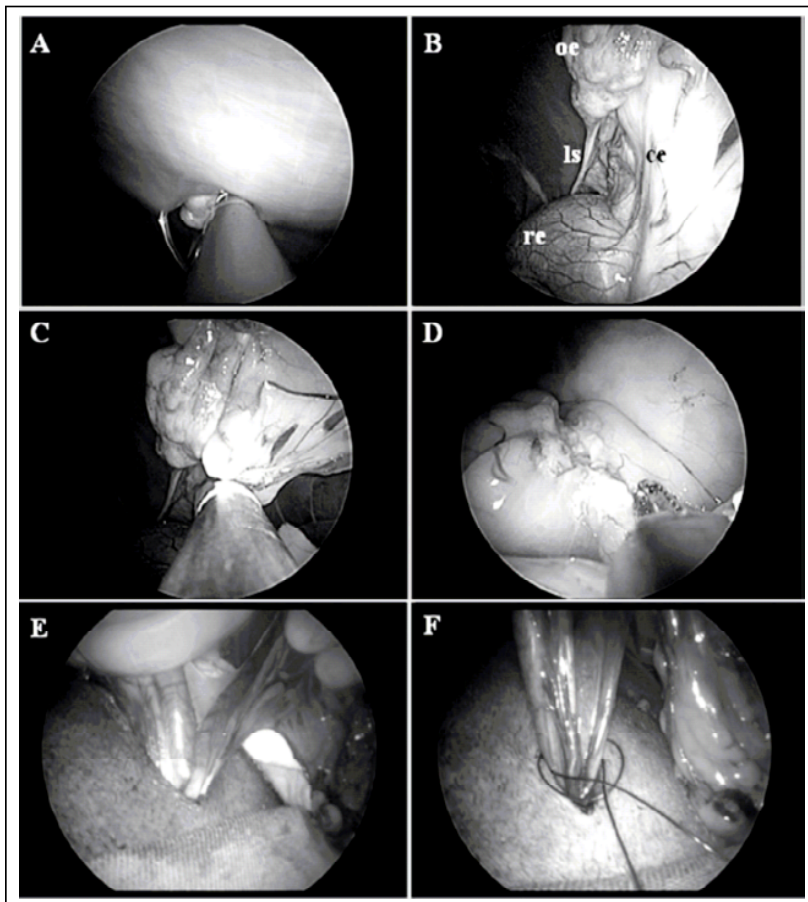

Figura 2 - Etapas cirúrgicas da SPVA-OSH. (A) Apreensão e suspensão do corno uterino esquerdo para passagem transparietal de fio de sutura de reparo; (B) exposição cirúrgica do pedículo ovariano esquerdo, observando-se o ligamento suspensor do ovário (ls), o ovário (oe), CAVO esquerdo (ce) e o rim esquerdos (re); (C) CAVO esquerdo sendo coagulado e seccionado com pinça de coagulação bipolar e corte ativo (Lina Tripol PowerBade ${ }^{\circledR}$ ); (D) pedículo ovariano totalmente coagulado e seccionado; (E) exteriorização dos cornos uterinos e da bifurcação uterina; (F) nó de Miller modificado, sendo aplicado cranial à cérvix.

bipolar. Empregou-se 40 watts de potência de coagulação bipolar com gerador de eletrocirurgia microprocessado ${ }^{c}$ (Figura 1C). Após coagulação e secção do CAVO esquerdo (Figuras $2 \mathrm{C}$ e D), a sutura de arrimo foi removida e a mesma manobra cirúrgica foi realizada do lado contra-lateral, para secção e hemostasia do pedículo ovariano direito.

Finalmente, o corno uterino direito foi apreendido e exteriorizado da cavidade abdominal, seguido pela exposição da bifurcação uterina e do corno uterino/ovário esquerdo (Figura 2E) e drenagem do pneumoperitônio. Caso o diâmetro dos ovários e dos cornos uterinos fossem desproporcionais à amplitude da miniceliotomia, a incisão seria ampliada em $1 \mathrm{~cm}$ no sentido craniocaudal para exteriorização adequada dos referidos órgãos. Para hemostasia do corpo uterino, empregou-se ligadura com nó de Miller modificado, posicionada cranial à cérvix, usando fio de poliglecaprone $25 \mathrm{n}^{\circ} 0$ (Figura $2 \mathrm{~F}$ ). Após a ressecção do útero, procedeu-se à omentalização e reposicionamento do coto uterino na cavidade abdominal. A laparorrafia foi realizada com único ponto em padrão Sultan, utilizando-se fio de poliglecaprone 25 no 0 , e a dermorrafia, em padrão Wolf, com náilon no3-0. Caso fosse necessária conversão do procedimento laparoscópico para laparotomia a fim de resolver possíveis complicações, a síntese seria realizada com os mesmos fios e padrões para laparorrafia, seguido por redução do espaço morto com fio de poliglecaprone 25 n3-3-0 e dermorrafia em padrão Wolf, com náilon no3-0.

Os cuidados pós-operatórios constaram de antibioticoterapia com penicilina benzatina $\left(40.000 \mathrm{UI} \mathrm{kg}^{-1}\right.$, duas administrações, IM, intervalo de 72 horas), analgesia com tramadol $\left(2 \mathrm{mg} \mathrm{kg}^{-1}, \mathrm{SC}\right.$, TID, durante três dias) e dipirona sódica ( $25 \mathrm{mg} \mathrm{kg}^{-1}$, VO, TID, durante três dias), além de higienização da ferida cirúrgica com gaze esterilizada e solução fisiológica. Os pontos foram 
removidos depois de decorridos oito dias do procedimento cirúrgico. Os animais foram devolvidos à APA após 24 horas do pós-cirúrgico.

O tempo cirúrgico foi dividido em seis momentos: (a) insuflação: tempo transcorrido entre a punção abdominal e o estabelecimento de pneumoperitônio; (b) acesso cirúrgico: período entre a incisão de pele à inserção do trocarte; (c) $C A V O$ esquerdo: tempo despendido na abordagem do pedículo ovariano esquerdo; (d) CAVO direito: idem ao pedículo esquerdo; (e) corpo uterino: período consumido para abordagem do corpo uterino; e (f) sintese abdominal: tempo despendido para laparorrafia e dermorrafia.

O tempo gasto em minutos em cada manobra cirúrgica foi avaliado pelo teste de múltiplas comparações de Bonferroni, após análise de variância de via única. Ademais, o tempo cirúrgico total entre as cinco primeiras, cinco segundas, quatro terceiras e cinco últimas cirurgias foi comparado pelos mesmos testes. Nível de significância de $\mathrm{P}<0,05$ foi adotado em todas as análises.

Para as possíveis complicações transcirúrgicas, empregou-se escala mista qualitativa/ quantitativa referente à hemorragia de um ou dos dois CAVOs, de coto uterino e resultante da lesão iatrogênica a outros órgãos. Considerou-se hemorragia leve de um ou mais CAVOs como perda sanguínea inferior a $5 \mathrm{ml}$, estimada visualmente, contornada com coagulação adicional; grau moderado, perda de 5 a $20 \mathrm{ml}$ de sangue, contornada com cauterização bipolar complementar; hemorragia grave, perda superior a $20 \mathrm{ml}$ de sangue, associado à necessidade de conversão/ laparotomia para controle adequado. Mesmo critério de classificação de hemorragia e necessidade de conversão para laparotomia foi adotado para o coto uterino e para outros órgãos que fossem lesionados acidentalmente. Os dados referentes às complicações trans-cirúrgicas foram analisados descritivamente. Os resultados são apresentados na forma de média e desvio padrão $( \pm \mathrm{DP})$.

\section{RESULTADOS E DISCUSSÃO}

A ovário-histerectomia vídeo-assistida, com único canal de trabalho, mostrou-se de fácil execução e com um tempo de procedimento relativamente curto. O tempo cirúrgico médio para a realização das SPVAOSHs, tomando-se em consideração todos os procedimentos realizados, foi de 22,95 $\pm 5,17$ (intervalo de 15,2 a 35,3 minutos) (Tabela 1). No entanto, DEVITT et al. (2005) descreveram um método simples de OSH vídeo-assistida em cadelas que durou 41 minutos em média. Além disso, os autores empregaram dois portais de $11 \mathrm{~mm}$, sendo um na região umbilical e o outro na região pré-púbica. MALM et al. (2004) empregaram quatro portais para realizar OSH total laparoscópica, sendo três trocartes de $11 \mathrm{~mm}$ e um de $6 \mathrm{~mm}$, e obtiveram tempo cirúrgico médio de $61,6( \pm 14,1)$ minutos. Esse tempo cirúrgico foi maior que o obtido no presente estudo. Acredita-se que tal diferença tenha ocorrido devido ao tipo de hemostasia dos pedículos ovarianos e do útero, que foi feito com clipes hemostáticos de titânio e endoloop, respectivamente, as quais são tecnicamente mais difíceis e consomem mais tempo cirúrgico que a coagulação bipolar com corte simultâneos (MALM et al., 2004). Ademais, o caráter vídeo-assistido da SPVA-OSH, ou seja, a coagulação e secção dos pedículos ovarianos e posterior exteriorização do corpo uterino pela miniceliotomia prépúbica para sua ligadura e secção extracorpórea conferiram maior agilidade em comparação às abordagens cirúrgicas, realizadas totalmente por laparoscopia com dois, três ou quatro portais (MALM

Tabela 1 - Tempo (minutos) das etapas intra-operatórias realizadas durante o procedimento de ovário-histerectomia vídeo-assistida com único portal (SPVA-OSH).

\begin{tabular}{llllllll}
\hline & Insuflação & $\begin{array}{c}\text { Acesso } \\
\text { cirúrgico }\end{array}$ & $\begin{array}{c}\text { CAVO } \\
\text { esquerdo }\end{array}$ & $\begin{array}{c}\text { CAVO } \\
\text { direito }\end{array}$ & $\begin{array}{c}\text { Corpo } \\
\text { Uterino }\end{array}$ & $\begin{array}{c}\text { Síntese } \\
\text { abdominal }\end{array}$ & Total \\
\hline Média \pm DP & $1,26 \pm 0,19$ & $0,60 \pm 0,16$ & $8,22 \pm 3,82^{\mathrm{a}}$ & $7,66 \pm 2,17^{\mathrm{a}}$ & $3,56 \pm 0,38^{\mathrm{b}}$ & $1,76 \pm 0,20$ & $22,95 \pm 5,17$ \\
Mínimo & 0,90 & 0,40 & 4,20 & 4,50 & 3,00 & 1,30 & 15,20 \\
Máximo & 1,50 & 0,90 & 18,30 & 12,50 & 4,20 & 2,30 & 35,30 \\
CV $(\%)$ & 17,47 & 27,24 & 46,47 & 28,36 & 10,67 & 33,60 & 23,13 \\
\hline
\end{tabular}

Insuflação: tempo compreendido entre a celiocentese com agulha de Veress e estabelecimento de pneumoperitônio de $10 \mathrm{mmHg}$; acesso cirúrgico: incisão de pele e introdução do trocarte; CAVO esquerdo/direito: abordagem cirúrgica aos pedículos ovarianos esquerdo e direito; corpo uterino: abordagem cirúrgica ao corpo uterino; síntese abdominal: laparorrafia e dermorrafia.

${ }^{a}$ Diferem significativamente $(\mathrm{P}<0,001)$ das demais etapas ao teste Bonferroni.

${ }^{b}$ Difere significativamente da insulflação $(\mathrm{P}<0,001)$ e da síntese abdominal $(\mathrm{P}<0,05)$ ao teste Bonferroni.

Ciência Rural, v.41, n.2, fev, 2011. 
et al., 2004; DEVITT et al., 2005; RUÍZ et al., 2008), sem, contudo, perder a característica de mínima invasão. MALM et al. (2004) complementaram que cadelas castradas por celiotomia mediana pré-retro-umbilical (grupo controle) foram operadas, em média, em 21,1 $( \pm 4,3)$ minutos. Esse tempo foi semelhante ao obtido no presente estudo, porém empregando-se a técnica de OSH vídeo-assistida com único portal (Tabela 1).

O tempo cirúrgico total gasto nas cinco primeiras, cinco segundas, quatro terceiras e cinco últimas cirurgias foi de, respectivamente, $29,16 \pm 4,27$, $21,74 \pm 2,54,23,33 \pm 3,14$ e 17,66 $\pm 2,34$ minutos, com tempo cirúrgico médio de 22,95 $\pm 5,17$ minutos. O tempo gasto nas cinco primeiras cirurgias foi significativamente mais longo que as cinco segundas $(\mathrm{P}<0,05)$ e que as cinco últimas cirurgias $(\mathrm{P}<0,001)$.

Esses resultados demonstraram que a obtenção de tempo cirúrgico médio inferior a 25 minutos pode ser alcançado após cinco procedimentos cirúrgicos, por um cirurgião apto a realizar procedimentos laparoscópicos, porém não proficiente na técnica de SPVA-OSH. Segundo RAHAL \& INÁCIO (1995), é esperado que o tempo cirúrgico seja maior nas primeiras etapas do treinamento em técnicas cirúrgicas laparoscópicas.

Considerando os 19 procedimentos realizados, as etapas que consumiram maior tempo cirúrgico $(\mathrm{P}<0,001)$ foram a manipulação, coagulação e secção dos pedículos ovarianos esquerdo $(8,2 \pm 3,7 \mathrm{~min})$ e direito $(7,7 \pm 2,1 \mathrm{~min})$. MALM et al. (2004) afirmaram que a hemostasia dos pedículos ovarianos e corpo uterino foram os momentos cirúrgicos que resultaram em maiores dificuldades técnicas, contribuindo sensivelmente para o alto tempo cirúrgico médio obtido $(61,6 \pm 14,1 \mathrm{~min})$ e acrescentaram que tais limitações estão diretamente relacionadas à curva de aprendizado. Todavia, não avaliaram objetivamente a curva de aprendizado, tampouco o tempo cirúrgico consumido em cada etapa intra-operatória.

Várias são as vantagens da realização da ovário-histerectomia vídeo-assistida com único portal sobre outros acessos cirúrgicos por celiotomia ou laparoscopia. A manobra de rotação do tronco no período trans-cirúrgico, conforme descrito por SILVA (2008), facilitou sobremaneira a exposição e coagulação/ secção dos CAVOs. Ademais, não foi necessário romper o ligamento suspensor do ovário para visualização do pedículo ovariano, o qual foi propriamente coagulado e seccionado sob visualização direta. A ruptura manual e às cegas do ligamento suspensor do ovário frequentemente se faz necessária para melhor acesso cirúrgico ao CAVO durante a ovário-histerectomia via celiotomia pré-retro-umbilical, sobretudo em cadelas de raças que apresentam tórax e/ou pedículos ovarianos profundos (STONE et al., 1998; FOSSUM, 2008). Ressalte-se que, devido à inserção do ligamento suspensor do ovário no peritônio, na região do pólo cranial do rim, sua ruptura é fonte de dor trans e pósoperatória, além de gerar hematomas retroperitoneais e riscos de ruptura parcial ou total dos CAVOs (BURROW et al., 2005).

O posicionamento estratégico do portal de $11 \mathrm{~mm}$ na região pré-pubica foi de extrema importância para a realização do procedimento vídeo-assistido, permitindo exteriorização adequada do corpo do útero, inclusive da cérvix, em cadelas pesando entre $8 \mathrm{e} 18 \mathrm{~kg}$. Ressalta-se que cinco cadelas eram pré-púberes, as quais não necessitaram ampliação do acesso cirúrgico para exteriorização da bifurcação do corpo do útero. Frequentemente, a ampliação da celiotomia no sentido craniocaudal se faz necessária quando o procedimento é realizado por celiotomia mediana pré-retro-umbilical em cadelas pré-púberes, devido ao posicionamento mais caudal da bifurcação uterina e da cérvix em animais mais jovens (STONE et al., 1998; MALM et al., 2004; BENCHARIF et al., 2010).

Com relação às complicações transoperatórias, 14 animais (70\%) não apresentaram complicações de nenhuma natureza. Em duas cadelas (10\%), houve punção acidental de baço com agulha de Veress durante o estabelecimento do pneumoperitônio com sangramento do parênquima esplênico, resultando em hemorragia grave e conversão para celiotomia. $\mathrm{Na}$ cadela número 14, foi possível realizar a SPVA-OSH, mesmo tendo sido constatada lesão iatrogênica do baço. Contudo, após algumas tentativas ineficazes de conter a hemorragia mediante compressão do foco da lesão com pinça de apreensão e turunda de gaze, foi necessário converter o procedimento para celiotomia, seguido de esplenectomia. Na cadela número 15, foi feita a conversão para laparotomia mediana, devido à presença de sangramento arterial esplênico, procedendo-se então à esplenectomia e ováriohisterectomia convencional, sendo esse animal excluído da avaliação estatística. Ambas as cadelas passaram pelos mesmos cuidados pós-operatórios instituídos para todas as demais e, apesar do quadro hemorrágico em ambas, não houve necessidade de hemoterapia e os pacientes convalesceram satisfatoriamente. Acredita-se que tais iatrogenias tenham sido influenciadas pelo protocolo anestésico empregado. Os fenotiazínicos são conhecidos por promoverem esplenomegalia, o que aumenta significativamente as chances de traumatismo ao baço, conforme mencionado por DAVIDSON et al. (2004). Os autores complementaram que obtiveram punção acidental de 
baço com agulha de Veress, posicionada na região umbilical (DAVIDSON et al., 2004). Ressalte-se que as punções esplênicas obtidas no presente estudo não resultaram da técnica de SPVA-OSH em si, mas sim do método de estabelecimento do pneumoperitônio. Acredita-se que, com o emprego da técnica aberta para criação do pneumoperitônio, o risco de lesão iatrogênica dos órgãos intra-abdominais seja sensivelmente minimizado.

Houve hemorragia de grau leve em apenas um dos pedículos ovarianos, em duas cadelas (10\%), que foi facilmente identificada e imediatamente controlada mediante coagulação complementar com pinça bipolar. Outras duas cadelas (10\%) apresentaram hemorragia de grau moderado após a coagulação/ secção de um dos CAVOs, que foram devidamente controladas após coagulação complementar com pinça bipolar. As hemorragias ocorreram pela secção dos CAVOs sem prévia coagulação satisfatória. Tal complicação foi atribuída à inexperiência do cirurgião durante os procedimentos. A curva de aprendizado para adquirir proficiência em determinada técnica cirúrgica laparoscópica específica pode necessitar dezenas de procedimentos para ser alcançada (MELO, 2004). Em um levantamento sobre colecistectomia, fundoplicatura e colectomia realizadas por cirurgiões não proficientes em cirurgia laparoscópica, constatou-se que a proficiência foi obtida após, respectivamente, 30,28 e 40 procedimentos (DAGASH et al., 2003). Dessa forma, acredita-se que para se adquirir menores taxas de complicações trans-cirúrgicas, sejam necessárias entre 30 e 40 SPVA-OSHs.

Apesar de não ter sido o principal objetivo do presente estudo, ressalta-se que os animais se recuperaram satisfatoriamente no período pós-cirúrgico imediato, retornando às atividades de deambulação e micção na primeira hora de pós-operatório, e defecação e ingestão de água e alimentos dentro das primeiras doze horas. Ademais, não foi empregado colar elizabetano em nenhuma cadela, não tendo havido complicações relacionadas à dor, deiscência e contaminação de ferida cirúrgica até o momento da retirada dos pontos cutâneos.

\section{CONCLUSÃO}

A SPVA-OSH foi realizada em tempo cirúrgico médio de 22,95 minutos e sem complicações graves em 19 de 20 cadelas operadas, por um cirurgião não proficiente na técnica. Os primeiros cinco procedimentos consumiram mais tempo cirúrgico que os demais e a abordagem aos pedículos ovarianos esquerdo e direito foram as etapas cirúrgicas que apresentaram maior tempo de execução. A taxa de complicações trans-operatórias foi de $30 \%$.

\section{AGRADECIMENTOS}

Os autores do presente estudo agradecem à $\mathrm{Sr}^{\mathrm{a}}$ - Vilma, Gerente Comercial da WEM (Ribeirão Preto - SP), pela concessão das pinças de coagulação bipolar, Lina Tripol PowerBlade $^{\circledR}$, empregadas no presente estudo.

\section{FONTES DE AQUISIÇÃO}

a - H. B. Strattner, Ribeirão Preto-SP.

b - Lina PowerBlade Tripol ${ }^{\circledR}$, WEM e Vivamed, Ribeirão Preto-SP.

c - HF- $120^{\circledR}$,WEM e Vivamed, Ribeirão Preto- SP.

\section{COMITÊ DE ÉTICA E BIOSSEGURANÇA}

O presente estudo foi aprovado pela Comissão de Ética no Uso de Animais (CEUA) da Faculdade de Ciências Agrárias e Veterinárias, Universidade Estadual Paulista (FCAV/ UNESP - Jaboticabal), protocolado sob o número 004731-10.

\section{REFERÊNCIAS}

BENCHARIF, D. et al. Ovariohysterectomy in the bitch. Obstetrics and Gynecology International, v.2010, p.1-7, 2010. Disponível em: <http://www.ncbi.nlm.nih.gov/pmc/ articles/PMC2833413/?tool=pubmed $>$. Acesso em: 9 out. 2010. doi: $10.1155 / 2010 / 542693$.

BRUN, M.V. et al. Ovário-histerectomia em caninos por cirurgia laparoscópica. Brazilian Journal of Veterinary Research and Animal Science (Online), v.37, n.6, 2000. Disponível em: <http:/ / w w w. scielo.br/s c i e lo.ph p ? pid=S 1413 $95962000000600011 \&$ script $=$ sci_abstract\&tlng=pt $>$. Acesso em: 9 out. 2010. doi: 10.1590/S1413-95962000000600011.

BURROW, R. et al. Complications observed during and after ovariohysterectomy of 142 bitches at a veterinary teaching hospital. Veterinary Record, v.157, p.829, 2005. Disponível em: <http://veterinaryrecord.bmj.com/content/ 157/26/829.full.pdf>. Acesso em: 9 out. 2010. doi: 10.1136/ vr.157.26.829.

DAGASH, H. et al. When can I be proficient in laparoscopic surgery? A systematic review of the evidence. Journal of Pediatric Surgery, v.38, p.720-724, 2003. Disponível em: < h t t p: // ww w.jpedsurg. org/article/S0022$3468 \% 2803 \% 2900036-8 /$ pdf $>$. Acesso em 9 out. 2010. doi: $10.1016 /$ jpsu.2003.50192.

DAVIDSON, E.B. et al. Comparison of laparoscopic ovariohysterectomy and ovariohysterectomy in dogs. Veteinary Surgery, v.33, n.1, p.62-69, 2004. Disponível em: <http:// on linelibrary.wiley.com/doi/ $10.1111 /$ j. 1532 950X.2004.04003.x/abstract>. Acesso em: 9 out. 2010. doi: 10.1111/j.1532-950X.2004.04003.x.

DEVITT, C.M. et al. Duration, complications, stress, and pain of open ovariohysterectomy versus a simple method of laparoscopicassisted ovariohysterectomy in dogs. Journal of the American Veterinary Medicine Association, v.227, n.6, p.921-927, 2005. Disponível em: <http://avmajournals.avma.org/doi/abs/10.2460/ 
javma.2005.227.921>. Acesso em: 9 out. 2010. doi: 10.2460/ javma.2005.227.921.

FREEMAN, L.J. Veterinary endosurgery. Saint Louis: Mosby, 1999. 276p.

FREEMAN, L.J. et al. Comparison of pain and postoperative stress in dogs undergoing natural orifice transluminal endoscopic surgery, laparoscopic, and open oophorectomy. Gastrointestinal Endoscopy (in press), 2010. Disponível em: <http://www.giejournal.org/article/ S0016-5107\%2810\%2900139-2/abstract>. Acesso em: 9 out. 2010 . doi: 10.1016/j.gie.2010.01.066.

FOSSUM, T.W. Cirurgia de pequenos animais. 3.ed. Rio de Janeiro: Guanabara Koogan. 2008. 1314p.

GOWER S, MAYHEW P. Canine laparoscopic and laparoscopic-assisted ovariohysterectomy and ovariectomy. Compendium: continuing education for veterinarians, v.30, n.8, p.430-440, 2008. Disponível em: <http:// tapgabjoypep.59.to/Media/Publications Article/ PV_30_08_430.pdf>. Acesso em: 9 out. 2010.

HANCOCK, R.B. et al. Comparison of postoperative pain after ovariohysterectomy by harmonic scalpel-assisted laparoscopy compared with median celiotomy and ligation in dogs. Veterinary Surgery, v.34, n.3, p.273-282, 2005. Disponível em: <http://onlinelibrary.wiley.com/doi/10.1111/ j.1532-950x.2005.00041.x/abstract>. Acesso em: 9 out. 2010. doi: $10.1111 / \mathrm{j} .1532-950 x .2005 .00041 . x$.

HOWE, M.L. Surgical methods of contraception and sterilization. Theriogenology, v.66, p.500-509, 2006. Disponível em: <http://www.theriojournal.com/article/S0093691X\%2806\%2900231-7/abstract>. Acesso em: 9 out. 2010. doi: $10.1016 /$ j.theriogenology.2006.04.005.

MALM, C. et al. Ovário-histerectomia: estudo experimental comparativo entre as abordagens laparoscópica e aberta na espécie canina. Intra-operatório - I. Arquivo Brasileiro de Medicina Veterinária e Zootecnia, v.56, n.4, p.457-466, 2004. Disponível em: <http://www.scielo.br/scielo.php?pid=S010209352004000400006\&script $=$ sci_abstract\&tlng=pt $>$. Acesso em: 9 out. 2010. doi: 10.1590/S0102-09352004000400006.

MELO, M.A.C. Curva de aprendizado na vídeocirurgia. Revista Brasileira de Videocirurgia, v.2, n.3, p.111-113, 2004. Disponível em: <http://www.ppge.ufrgs.br/giacomo/arquivos/ eco02277/melo-2004.pdf>. Acesso em: 9 out. 2010.

RAHAL, F.; INÁCIO, V. Treinamento em videocirurgia. In: COELHO, J.C.U. et al. (Eds.). Complicações da videocirurgia: da profilaxia ao tratamento. Rio de Janeiro: MEDSI, 1995. p.11-16.

RUÍZ, I.C. et al. Descripción y evaluación de una técnica de ovariohisterectomía laparoscópica en perras sanas. Revista Colombiana de Ciências Pecuárias, v. 21, p.546-558, 2008. Disponível em: <http://rccp.udea.edu.co/index.php/ojs/article/ view/366/0>. Acesso em: 9 out. 2010.

SILVA, M.A.M. Avaliação laparoscópica das aderências intraperitoneais pós-cirúrgicas em cadelas: emprego de duas doses de solução de azul de metileno a $1 \%$ na profilaxia. 2008. 74f. Dissertação (Mestrado em Ciência Animal), Programa de Pós-Graduação em Ciência Animal, Universidade Federal de Goiás, GO.

STONE, E.A. et al. Ovário e útero. In: SLATER, D. (Ed.). Manual de cirurgia de pequenos animais. 2.ed. São Paulo: Manole, 1998. V.2, p.1540-1558.

VAN NIMWEGEN, S.A.; KIRPENSTEIJN, J. Comparison of Nd:YAG surgical laser and remorgida bipolar electrosurgery forceps for canine laparoscopic ovariectomy. Veterinary Surgery, v.36, p.533-540, 2007. Disponível em: <http:// on linelibrary.wiley.com/doi/10.1111/j. 1532 950X.2007.00304.x/abstract>. Acesso em: 9 out. 2010. doi: 10.1111/j.1532-950X.2007.00304.x. 